Article

\title{
miR-361-5p Mediates SMAD4 to Promote Porcine Granulosa Cell Apoptosis through VEGFA
}

\author{
Mengnan Ma, Jinbi Zhang, Xiaomeng Gao, Wang Yao, Qifa Li $@$ and Zengxiang Pan * \\ College of Animal Science and Technology, Nanjing Agricultural University, Nanjing 210095, China; \\ 18795975502@163.com (M.M.); zhangjinbi@njau.edu.cn (J.Z.); 2016105013@njau.edu.cn (X.G.); \\ njauyw@163.com (W.Y.); liqifa@njau.edu.cn (Q.L.) \\ * Correspondence: owwa@njau.edu.cn
}

Received: 29 July 2020; Accepted: 3 September 2020; Published: 4 September 2020

\begin{abstract}
Follicular atresia is an inevitable degenerative process that occurs in mammalian ovarian follicles. The molecular events involved in atresia, particularly granulosa cell apoptosis, have long attracted researchers' attention. Vascular endothelial growth factor A (VEGFA) is downregulated during follicular atresia in porcine ovaries and serves as an inhibitor of apoptosis in granulosa cells. In addition, transforming growth factor (TGF)- $\beta$ signaling has been considered a central trigger in granulosa cell apoptosis. However, the link between TGF- $\beta$ signaling and VEGFA is unknown. We proved that miR-361-5p is significantly upregulated during the atresia process and that it promotes GC apoptosis by directly targeting the VEGFA 3'UTR. In addition, we revealed that the miR-361-5p coding gene MIR361 was significantly downregulated by SMAD4, the central intracellular mediator of TGF- $\beta$ signaling, that bound to the MIR361 promoter. In conclusion, our findings expanded what is known about VEGFA posttranscriptional regulation and revealed a complete SMAD4/miR-361-5p/VEGFA regulatory network in ovarian granulosa cell apoptosis. These data provide useful references for follicular atresia and ovarian physiological function studies.
\end{abstract}

Keywords: GC apoptosis; VEGFA; miR-361-5p; TGF- $\beta$ signaling; SMAD4; follicular atresia

\section{Introduction}

Follicular atresia is a common physiological phenomenon that can occur at all stages of follicular development in mammals. At puberty, the porcine primordial follicle reserve contains approximately five million primordial follicles, most of which go through the atresia process when they become larger than $1 \mathrm{~mm}$ in diameter, which occurs during the antral stage. The atresia rate remarkably increases in antral follicles of approximately $3-5 \mathrm{~mm}$ in diameter [1]. Ultimately, the majority of follicles disappear before maturation, and less than $14 \%$ are ovulated [2].

Adequate blood flow is necessary for oxygen and nutrient supply in the ovary and is possibly a rate-limiting step in the selection and maturation of dominant follicles destined for ovulation [3]. The vascular endothelial growth factor (VEGF) family, which is composed of at least six members (VEGF A-F), is involved in the formation of blood vessels. VEGFA was the first VEGF member identified, and it is the most widely studied molecule; it is primarily known to be a factor that promotes angiogenesis and vasculogenesis [4]. VEGFA functions through its major tyrosine kinase receptor VEGFR2 (also known as kinase insert domain receptor, KDR) [5]. The expression and function of VEGFA have been studied in mammalian ovaries during follicular development. In humans, VEGF mRNA and protein are present in the theca layer and in granulosa cells (GCs) during antral follicle stages, which is when the vascular network is actively developing [6]. In bovines, VEGFA mRNA is expressed in both the theca interna and GCs and the VEGF protein level increases as the developmental stages of follicle growth progress [7]. In pigs, the expression levels of two VEGFA isoforms (VEGF120 
and VEGF164) in GCs and the levels of two receptors (VEGFR1 and VEGFR2) in theca cells appear to be higher in follicles of medium and large size than they are in small follicles [8]. VEGF production by granulosa cells was also proved to play a role in aortic endothelial cell proliferation, thus drive angiogenesis in the three-dimensional fibrin gel $[9,10]$. According to our earlier study in pigs, VEGFA mRNA levels were significantly decreased in early atretic follicles compared to healthy follicles [11], which implied a potential role of VEGFA during follicular atresia.

Regarding the transcriptional regulation of VEGFA, transcription factors, including hypoxiainducible factor (HIF) [12], estrogen receptors $\alpha$ and $\beta$ (ER $\alpha$ and $\beta$ ) [13], signal transducer and activator of transcription 3 (STAT-3), and Wilms tumor 1 (WT1) [14] were identified to regulate VEGFA through direct promoter binding. In recent years, an increasing number of studies have focused on the negative posttranscriptional regulation of VEGFA by miRNAs. Direct binding microRNAs such as miR-26a [15], miR-93 [16], miR-134 [17], miR-195 [18], miR-203 [19], miR-361-5p [20], and miR-503 [21] to the VEGFA $3^{\prime}$ UTR has been observed in a variety of cells and conditions, mostly in carcinoma. However, despite its key function in the ovary, the posttranscriptional regulation of VEGFA in ovarian follicles, especially during atresia, is still unknown.

It has been reported that the canonical transforming growth factor (TGF)- $\beta$ signaling pathway is involved in the proliferation and apoptosis of ovarian granulosa cells [22]. In our previous studies, SMAD family member 4 (SMAD4), the terminal regulatory molecule in TGF- $\beta$ signaling, was identified as an anti-apoptosis factor in granulosa cells [23]. Interestingly, this function of SMAD4 was found to be related to its direct binding to the promoters of a few miRNA coding genes to mediate the negative regulation of miRNA expression [24], which implied a possible regulatory axis involving SMAD4-miRNA-functional genes in GCs. Thus, we performed this study to identify a potential SMAD4/miR-361-5p/VEGFA regulatory axis in porcine GCs and proved that during follicular atresia, miR-361-5p significantly increases and promotes GC apoptosis by directly targeting the VEGFA $3^{\prime} \mathrm{UTR}$. The expression of the miR-361-5p coding gene MIR361 is negatively regulated by the SMAD4 binding. The study not only filled the gap in knowledge regarding miRNA-mediated regulation of VEGFA during follicular atresia but also provided evidence for the anti-apoptotic role of SMAD4 through the transcriptional regulation of miRNAs in GCs.

\section{Materials and Methods}

\subsection{Follicle Collection}

Ovaries were obtained from seven-month old unstimulated commercial replacement large white gilts at a local slaughterhouse. The ovaries were quickly washed twice with $75 \%$ ethanol and physiologic saline, and then individual antral follicles, approximately 3 to $5 \mathrm{~mm}$ in diameter, were dissected using small scissors and fine forceps and then classified as healthy follicles (HFs) and atretic follicles (AFs) according to follicle shape, GC density, and hormone levels [11]. Briefly, HFs are round with a sharp and continuous granulosa cell membrane, fixed, and visible cumulus-oocyte complex (COC), fine capillary vessels, and clear follicular fluid; HFs may have visible COC, but show gaps in membrane granulosa cells, less capillary vessels and turbid follicular fluid. Follicular components were then separated to further confirm the classification by detecting the ratio of progesterone and $17 \beta$-estradiol level (P4/E2) and the antral GC density. Follicles with a P4/E2 ratio of $<5$ were classified as HFs and a ratio of 5 to 20 as AFs. Densities of $<250$ cells $/ \mu$ L were classified as HFs, $250-1000$ cells $/ \mu \mathrm{L}$ were classified as AFs. Animal Ethics Committee of Nanjing Agricultural University, Nanjing, Jiangsu, China (SYXK 2017-0027)

\subsection{Cell Culture and Transfection}

Primary GCs were obtained from HFs by extracting via a syringe with a 20-gauge needle. The COC and ovarian tissue were discarded under a stereo microscope. GCs were then cultured with DMEM/F-12 medium (Invitrogen, Carlsbad, CA, USA) containing 10\% fetal bovine serum (Invitrogen, Carlsbad, 
CA, USA), 100 units $/ \mathrm{mL}$ penicillin and $100 \mathrm{mg} / \mathrm{mL}$ streptomycin at $37{ }^{\circ} \mathrm{C}$ in $5 \% \mathrm{CO}$. HEK293 cells were maintained in DMEM medium (Sigma, St. Louis, MO, USA) with 10\% fetal bovine serum ate the same condition. The VEGFA siRNA, Smad4 siRNA, miR-361-5p mimic, and miR-361-5p inhibitor, and their corresponding control oligos were synthesized by GenePharma (Shanghai, China) (Supplementary Table S1). For transfection porcine GCs were culture for $12 \mathrm{~h}$, then transfected with the appropriate plasmids or oligos using Lipofectamine 2000 and Opti-MEM (Invitrogen, Carlsbad, CA, USA), according to the manufacturer's protocol.

\subsection{Immunohistochemical Assay}

To examine the expression and location of VEGFA in healthy and atretic follicles, immunohistochemical staining was performed according to our previous description [25]. Rabbit polyclonal VEGFA (diluted 1:200 in PBS containing 1\% (w/v) bovine serum albumin, ab9570, Abcam, Cambridge, MA, USA) and a secondary antibody (G1210-2-A, Servicebio, Wuhan, China) were incubated with the slides. Specific protein immunoreactivity was visualized by incubation with $0.05 \%$ 3,30-diaminobenzidine (DAB, G1211, Servicebio, Wuhan, China) for $15 \mathrm{~min}$, and the slides were counterstained with haematoxylin (G1004, Servicebio, Wuhan, China). Images were captured under a microscope (Nikon Eclipse E200, Tokyo, Japan).

\subsection{RNA Extraction and $q R T-P C R$}

Total RNA was extracted from follicles and GCs using TRIzol reagent (Invitrogen, Carlsbad, CA, USA). Then, the extracted total RNA was reverse transcribed to generate cDNA using a Super M-MLV RTase Synthesis Kit, and qRT-PCR was performed using SYBR Premix Ex Taq (Takara, Dalian, China) on an ABI StepOne system (Applied Biosystems, Carlsbad, CA, USA), according to the manufacturer's instructions. GAPDH was used as an internal control. For miRNA detection, first-strand cDNA synthesis and qRT-PCR were performed using miRNA two-step qRT-PCR SuperMix (TransGen, Beijing, China). U6 was used as an internal control. For each gene primer set, a control containing no cDNA was included, and each reaction was repeated three times for every sample. The primers for qRT-PCR are listed in Supplementary Table S2.

\subsection{Fluorescent In Situ Hybridization (FISH)}

A FAM-labelled probe (5'-GTACCCCTGGAGATTCTGATAA-3') was specifically synthesized for miR-361-5p, and DAPI was used to stain the cell nuclei. GCs were cultured on coverslips, fixed in $4 \%$ paraformaldehyde (containing DEPC) for $20 \mathrm{~min}$, washed while shaking with PBS (pH 7.4) three times, and proteinase $\mathrm{K}(20 \mu \mathrm{g} / \mathrm{mL})$ was finally added for $5 \mathrm{~min}$ for digestion. Then, all procedures were conducted according to the manufacturer's instructions (Sevicebio, Wuhan, China). Finally, the images were acquired on a Nikon upright fluorescence microscope (Nikon DS-U3, Tokyo, Japan). Each experiment was performed three times.

\subsection{Protein Extraction and Western Immunoblotting Analysis}

GCs were washed with cold PBS and lysed with RIPA buffer containing $1 \%$ phosphatase inhibitor $(v / v)$ (Beyotime, Shanghai, China) and proteinase inhibitor (Sigma, St. Louis, MO, USA). The protein concentration was determined with a BCA Protein Assay Kit (Beyotime, Shanghai, China), and samples were diluted to the same concentration using $5 \times$ Protein Loading Dye (Sangon, Shanghai, China). Total protein extracts were separated by SDS-PAGE on $12 \%$ gels. The proteins were then transferred onto PVDF membranes (Millipore, Billerica, MA, USA), and the membranes were blocked with 5\% non-fat milk for $2 \mathrm{~h}$. After washing with Tris-buffered saline with Tween (TBST) for $15 \mathrm{~s}$, the membranes were incubated overnight at $4{ }^{\circ} \mathrm{C}$ with anti-VEGFA (diluted 1:5000, ab9570, Abcam, Cambridge, MA, USA), anti-Tubulin (diluted 1:1000, 10094-1-AP ProteinTech, Nanjing, China), and anti-CASP3 (diluted 1:1000, 19677-1-AP, ProteinTech, Nanjing, China). Then, the cells were incubated with a secondary peroxidase-conjugated antibody (diluted 1:2000, Cell Signaling Technology, Beverly, MA, USA) for 
$1 \mathrm{~h}$ at room temperature. Chemiluminescence was detected by WesternBright ${ }^{\mathrm{TM}} \mathrm{ECL}$ (Advansta, Menlo Park, CA, USA) and analyzed using the ImageJ software (Version 1.51w). Each experiment was performed three times.

\subsection{Plasmid Construction}

VEGFA 3'UTR fragments containing putative target sites for miR-361-5p and the promoter fragments of the miR-361-5p coding gene (MIR361) containing putative SMAD4 binding sites were amplified from porcine genomic DNA and were verified by sequencing. The VEGFA 3'UTR fragment was then digested with Nhel and Xbal (Thermo, Waltham, MA, USA) and cloned into a pmirGLO Dual-Luciferase miRNA Target Expression Vector (Promega Corporation, Madison, WI, USA). The MIR361 promoter fragment was digested with NheI and SacI, and then cloned into a pLL-3 reporter vector (Promega, Madison, WI, USA). The miR-361-5p plasmids with a mutated putative binding site were generated by the ClonExpress Entry One Step Cloning Kit (Vazyme, Nanjing, China), according to the manufacturer's protocol. Successful mutations were confirmed by sequencing. The overexpression plasmid pcDNA3.1-SMAD4 was generated previously by our group [26]. The primers used here are detailed in Supplementary Table S3.

\subsection{Luciferase Reporter Assays}

After a transfection period of $24 \mathrm{~h}$, the cells and lysates were collected. A Dual-Luciferase Reporter Assay System (Promega Corporation, Madison, WI, USA) was used to quantify luciferase activities following the manufacturer's instructions. Firefly luciferase activity was normalized to Renilla luciferase activity. Each experiment was performed six times.

\subsection{Apoptosis Assay}

GC apoptosis was measured with an annexin V-FITC/PI staining assay (Vazyme, Nanjing, China) according to the manufacturer's protocol. A cell-counting machine (Becton Dickinson, Franklin Lakes, NJ, USA) was used for the detection of apoptotic cells based on the principle of fluorescence-activated cell sorting (FACS). The data were analyzed using the FlowJo v7.6 software (Stanford University, Stanford, CA, USA).

\subsection{Statistical Analysis}

All data are presented as the means \pm S.E.M. The Prism 5 software (GraphPad Software) was used to perform the statistical analysis. Two-tailed Student's $t$-tests were used to evaluate the significance when two groups were compared. When three or more groups were compared, a one-way analysis of variance test was performed, and Tukey's test was used to determine significance between groups. $p$-values of $<0.05$ and 0.01 were considered to indicate significant and extremely significant differences, respectively.

\section{Results}

\subsection{VEGFA Is Downregulated in Atretic Follicles}

To investigate the VEGFA level during follicular atresia, we first determined the location of VEGFA in antral follicles by immunohistochemistry. The results showed a positive reaction in both theca and granulosa cells, and the brown staining was stronger in HFs (GCs closely arranged) than in AFs (GCs loosely arranged and partially dropped into the follicular cavity) (Figure 1A-D). In addition, mRNA levels of VEGFA detected in the whole follicle, GCs, and theca cells (TCs) by GeneChip Porcine GenomeArray (detailed data are contained in reference [11]) or qRT-PCR also suggested a significantly higher expression in HFs than in AFs (Figure 1E-G). ELISA for VEGFA showed a slight but significant decrease in VEGFA content in follicular fluid throughout the atresia process (Figure $1 \mathrm{H}$ ). These results suggested that VEGFA decreases during porcine follicular atresia. 

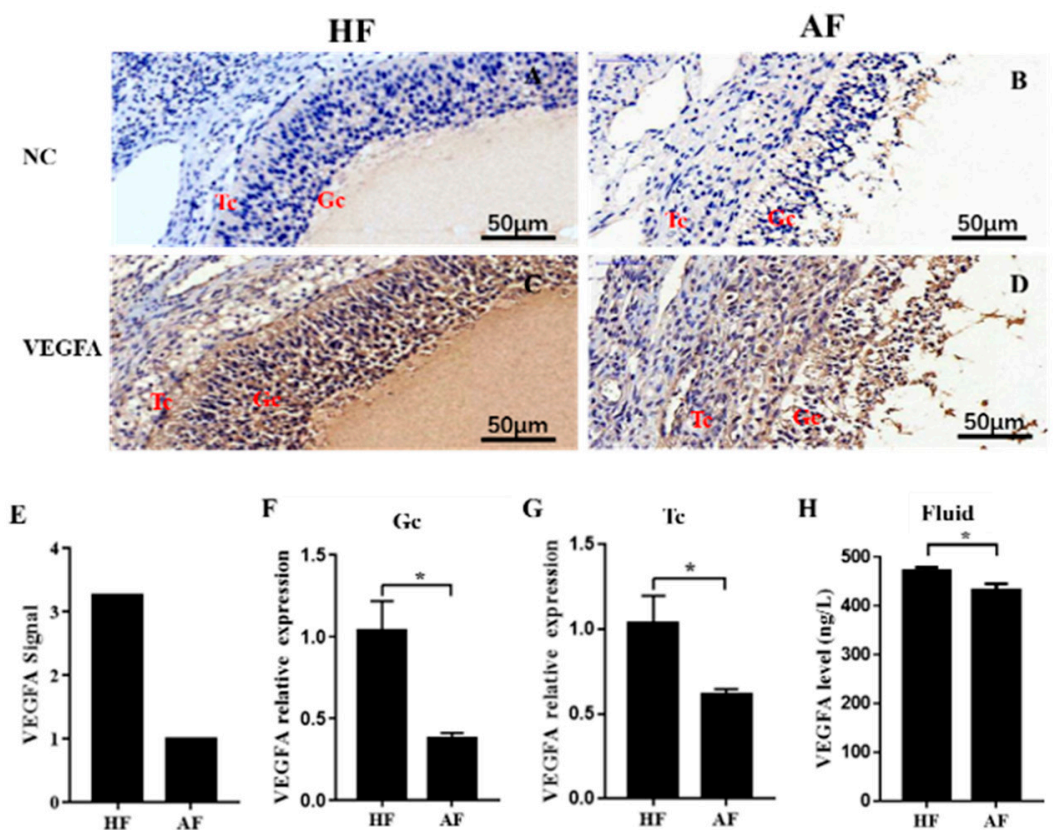

Figure 1. Expression of VEGFA in healthy and atretic antral follicles. (A-D): Immunolocalization of VEGFA in healthy $(\mathbf{A}, \mathbf{C})$ and atretic $(\mathbf{B}, \mathbf{D})$ antral follicles; $€$ : The signal intensity of VEGFA in follicles detected by the GeneChip Porcine Genome Array; $(\mathbf{F}, \mathrm{G})$ : relative expression levels of VEGFA in GC and TC, respectively, detected by qRT-PCR; $(\mathbf{H})$ : expression level of VEGFA in the follicle fluid detected by ELISA. NC, negative control; HF, healthy follicle; AF, atretic follicle; GC, granulosa cell; Tc, theca cell; scale bar $=50 \mu \mathrm{m}$. Data are expressed as the mean \pm SEM. ${ }^{*} p<0.05$.

\section{2. miR-361-5p Is Upregulated in Atretic Follicles}

To investigate the possible function of miR-361-5p during follicle atresia, we detected its location and expression levels in healthy and atretic follicles. FISH results showed that miR-361-5p was distributed in both TCs and GCs, and the signal was stronger in atretic follicles than in healthy follicles (Figure 2A-F). Further qualitative measurements were collected from the whole follicle, GCs, TCs, and follicular fluid by the microchip (data extracted from the $\mu$ Paraflo ${ }^{\mathrm{TM}}$ microfluidic chip used in our previous study for miRNA expression profiles in healthy and atretic follicles [27]) or qRT-PCR, respectively; those results also suggested significantly higher expression of mir-361-5p in atretic follicles than in healthy follicles in each of the follicle compartments (Figure 2G-J). These results implied that miR-361-5p was involved in the atresia process and may be involved in the posttranscriptional regulation of functional genes during follicular atresia.

\section{3. miR-361-5p Regulates VEGFA by Directly Binding to Its $3^{\prime} U T R$}

To further investigate the possible function of miR-361-5p in the regulation of VEGFA expression, the direct targeting of VEGFA by miR-361-5p was first predicted by bioinformatic methods and was confirmed by the luciferase reporter assay (Figure 3A). Next, we cultured porcine GCs transfected with miR-361-5p mimics or an inhibitor and then detected the mRNA/protein levels of VEGFA both in GCs and in the culture media. The results showed that both mRNAs (Figure 3B,C), and protein (Figure 3D,E) levels of VEGFA were significantly decreased after transfection with miR-361-5p mimics but increased after transfection with a miR-361-5p inhibitor (Figure 3B-E). In addition, we noticed that the levels of secreted VEGFA in culture media were slightly increased after transfection with a miR-361-5p inhibitor (Figure 3F,G). These results suggested that miR-361-5p negatively affected VEGFA expression by directly binding to its $3^{\prime}$ UTR in porcine GCs. 

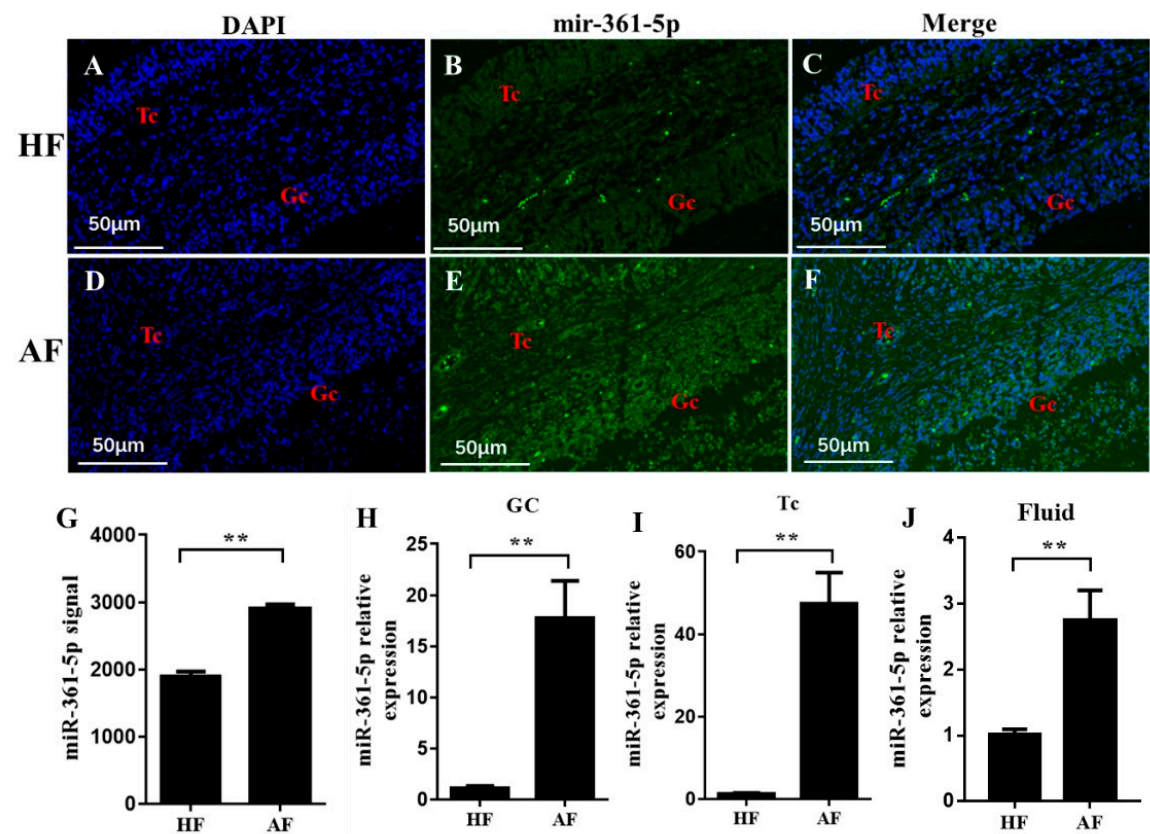

Figure 2. Expression of the mir-361-5p in healthy and atretic antral follicles. (A-F): RNA-FISH was utilized to examine the localization of mir-361-5p in healthy and atretic antral follicles; (G): Signal intensity of mir-361-5p in follicles detected by $\mu$ Paraflo $^{\mathrm{TM}}$ microfluidic chip; $(\mathbf{H}-\mathbf{J})$ : relative expression levels of mir-361-5p in GC, TC, and follicle fluid, respectively, detected by qRT-PCR. HF, healthy follicle; $\mathrm{AF}$, atretic follicle; GC, granulosa cell; $\mathrm{TC}$, theca cell; scale bar $=50 \mu \mathrm{m}$. Data are expressed as the mean \pm SEM. ${ }^{* *} p<0.01$.

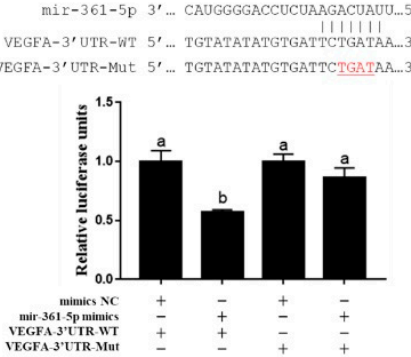

B
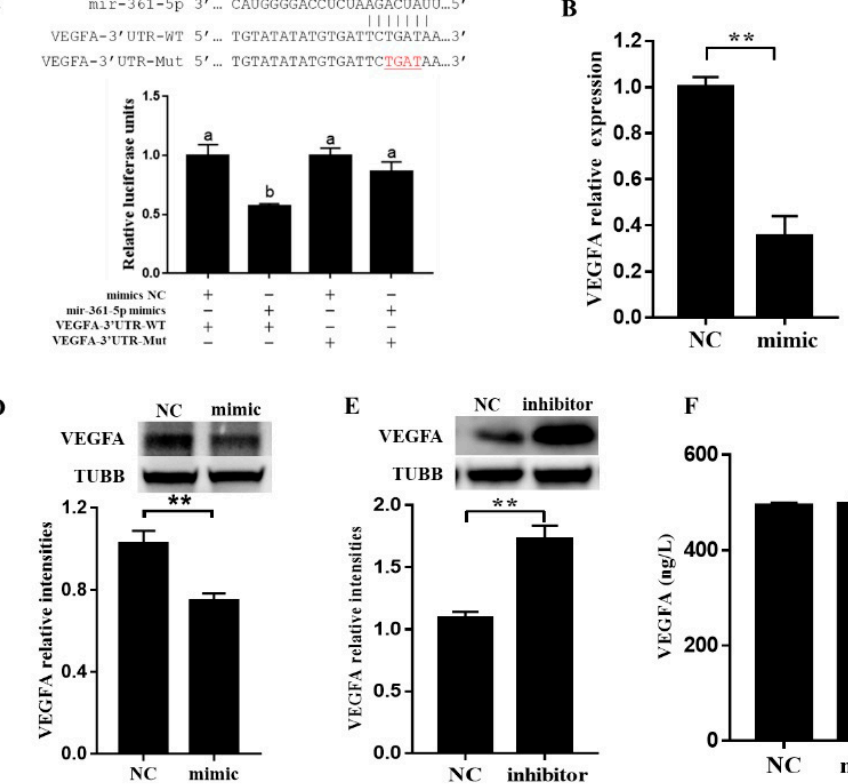

$\mathbf{E}$

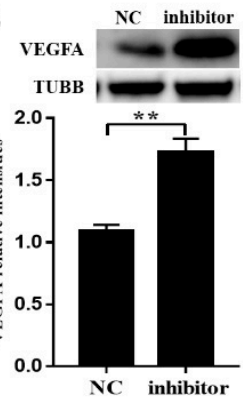

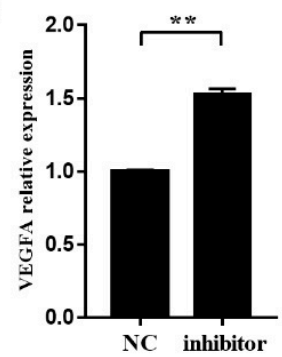

G

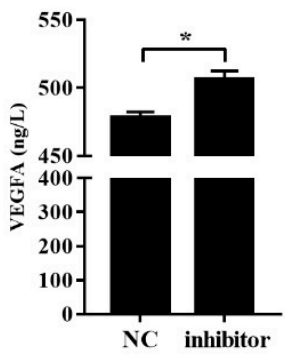

Figure 3. Negative regulation of VEGFA by miR-361-5p through direct 3'UTR binding. (A): The direct targeting of VEGFA by miR-361-5p confirmed by luciferase reporter assay in 293 cells (plus (+) indicates addition of oligos, minus (-) indicates no addition of oligos); (B,C): The mRNA levels of VEGFA after transfection of miR-361-5p mimics or inhibitors in porcine GCs; (D,E): The protein levels of VEGFA after transfection of miR-361-5p mimics or inhibitor in porcine GCs; (F,G): The levels of VEGFA after transfection of miR-361-5p mimics and inhibitors in culture media. $n=3$ cell culture wells per group. Data are expressed as the mean \pm SEM. Significant differences $(p<0.05)$ are indicated by different letters or ${ }^{*} p<0.05,{ }^{* *} p<0.01$. 


\section{4. miR-361-5p Regulates GC Apoptosis through VEGFA}

To determine whether miR-361-5p affects apoptosis of porcine GCs via regulation of VEGFA, we co-transfected a miR-361-5p inhibitor with a VEGFA siRNA. The FACS results suggested that the apoptosis rate was significantly decreased after miR-361-5p inhibitor transfection but reversed after the addition of VEGFA siRNA (Figure 4A). The protein levels of active cleaved Caspase 3 (c-CAS3) also showed a similar pattern (Figure 4B). Thus, miR-361-5p can promote GC apoptosis through VEGFA.

A
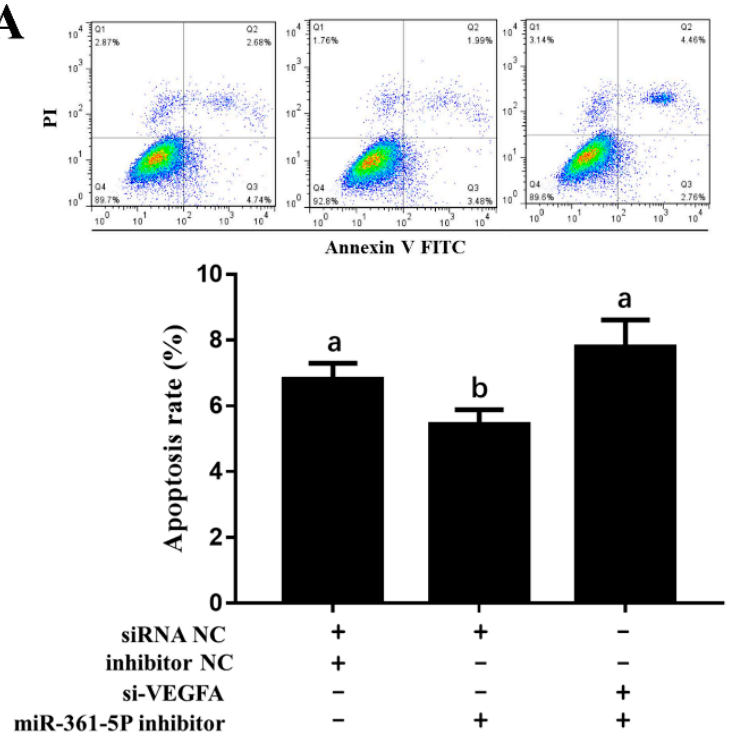

B
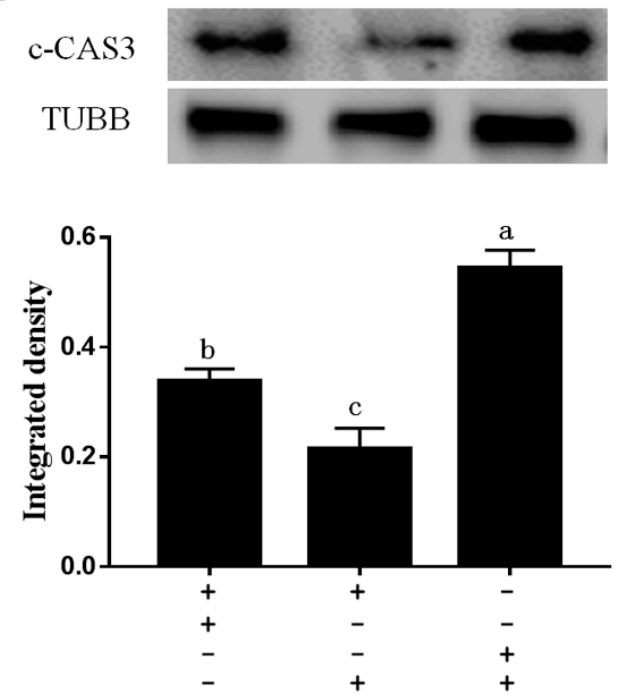

Figure 4. miR-361-5p regulates GC apoptosis through VEGFA. (A): GC apoptosis rate decreased after the transfection of miR-361-5p inhibitor and reversed after the addition of VEGFA siRNA detected by FACS analysis. (B): The protein levels of cleaved caspase 3 (c-CAS3) was down-regulated after the transfection of the miR-361-5p inhibitor and reversed after the addition of VEGFA siRNA. Plus (+) indicates addition of siRNAs or inhibitors, minus (-) indicates no addition of siRNAs or inhibitors. Data are expressed as the mean \pm SEM. Significant differences $(p<0.05)$ are indicated by different letters.

\subsection{SMAD4 Involved in miR-361-5p-Mediated VEGFA Expression}

To examine whether the expression of miR-361-5p was controlled by the TGF- $\beta$ signaling pathway, we analyzed the promoter region of the miR-361-5p coding gene MIR361 for potential binding sites. We identified four SMAD-binding elements (SBEs) within the region (Figure 5A). Next, we confirmed the role of the SBEs in MIR361 promoter activity with using a dual-luciferase reporter assay (Figure 5B). To further investigate the effect of SMAD4 on the MIR361 promoter, the increase and knockdown of SMAD4 were achieved by transfecting cells with a SMAD4 overexpression plasmid and siRNA, respectively. The luciferase reporter assay results suggested that SMAD4 had a negative effect on the MIR361 promoter (Figure 5C,D). In addition, in cultured porcine GCs, overexpression of SMAD4 did not show an apparent effect on miR-361-5p, but knockdown of SMAD4 significantly enhanced miR-361-5p expression (Figure 5E,F). Finally, both qRT-PCR and WB data suggested that knockdown of SMAD4 resulted in a significant decrease in VEGFA expression (Figure 5G,H). These results indicated that SMAD4 functioned as a trans-acting element to negatively regulate MIR361 transcription, and thereby, adjust VEGFA expression. 


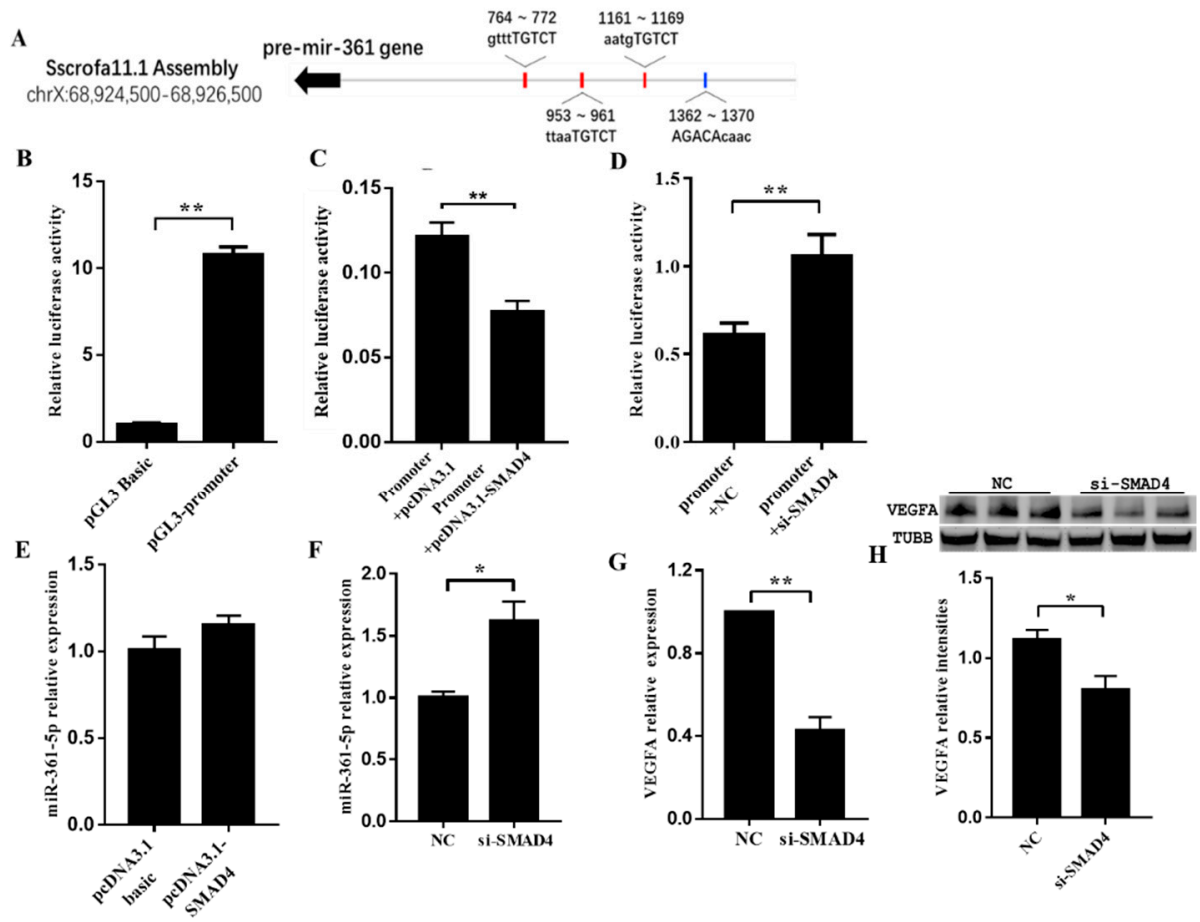

Figure 5. Transcription factor SMAD4 was involved in miR-361-5p mediated VEGFA expression. (A): Schematic diagram showing the genome location of the miR-361-5p coding gene and potential SMAD4 binding sites. (B): The promoter activity of MIR361 upstream region confirmed by the dual-luciferase reporter assay; (C,D): Overexpression and knockdown of SMAD4 weaken and enhanced MIR361 promoter activity, respectively; (E): The expression of miR-361-5p was not affected after SMAD4 overexpression; (F): The expression of miR-361-5p was up-regulated after SMAD4 knockdown; $(\mathbf{G}, \mathbf{H})$ : The expression of VEGFA mRNA and protein levels were down-regulated after SMAD4 knockdown. $n=3$ cell culture wells per group. Data are expressed as the mean \pm SEM. ${ }^{*} p<0.05,{ }^{* *} p<0.01$.

\section{Discussion}

miRNAs regulate gene expression by binding to specific sequences in target mRNAs, resulting in transcriptional repression [28] or degradation [29] of target mRNAs. In the field of reproduction, the critical roles of miRNAs in ovarian function, follicle development, and luteal formation have continually attracted attention and were recently connected to GC apoptosis and follicular atresia processes in humans, mice, bovines and pigs [30]. Our previous study, which compared the differential expression of miRNAs in healthy and atretic follicles, suggested a significant increase in miR-361-5p during atresia [27]. The fact that miR-361-5p was found to inhibit cell proliferation and metabolism and induce apoptosis in many cancer studies [31-33] also implied a possible relationship between miR-361-5p and GC apoptosis during the atresia process. In this study, we confirmed a direct interaction between miR-361-5p and VEGFA, which is mainly produced in GCs, and plays essential roles in angiogenesis, GC function, and oocyte development in antral follicles [25]. In addition, our study explored the transcriptional regulation of the miR-361-5p coding gene MIR361 by SMAD4 and identified a straightforward regulatory network of SMAD4/miR-361-5p/VEGFA. Briefly, miR-361-5p reduces VEGFA mRNA expression by direct binding, thus promoting GC apoptosis. Further, SMAD4 increases VEGFA levels through negative regulation of MIR361 expression through promoter binding at the transcription level Figure 6.

It is well known that the expression of VEGFA could be regulated at the transcriptional level by several cis-acting mechanisms and factors such as HIF [12], ER $\alpha, \beta$ [13], STAT-3, and WT1 [14]. The direct regulation of VEGFA by TGF- $\beta$ signaling was reported to be carried out by SMAD3 binding. For example, in vascular smooth muscle cells, TGF- $\beta$ led to the formation of a complex of SMAD3 and HIF-1 $\alpha$ that, in turn, activated the VEGFA transcription [34]. SMAD4, on the other hand, is a universal 
mediator that plays a role in canonical TGF- $\beta$ signal transduction into the nucleus, where SMAD4 complexes regulate gene transcription positively or negatively with different coactivator or corepressor factors [35].

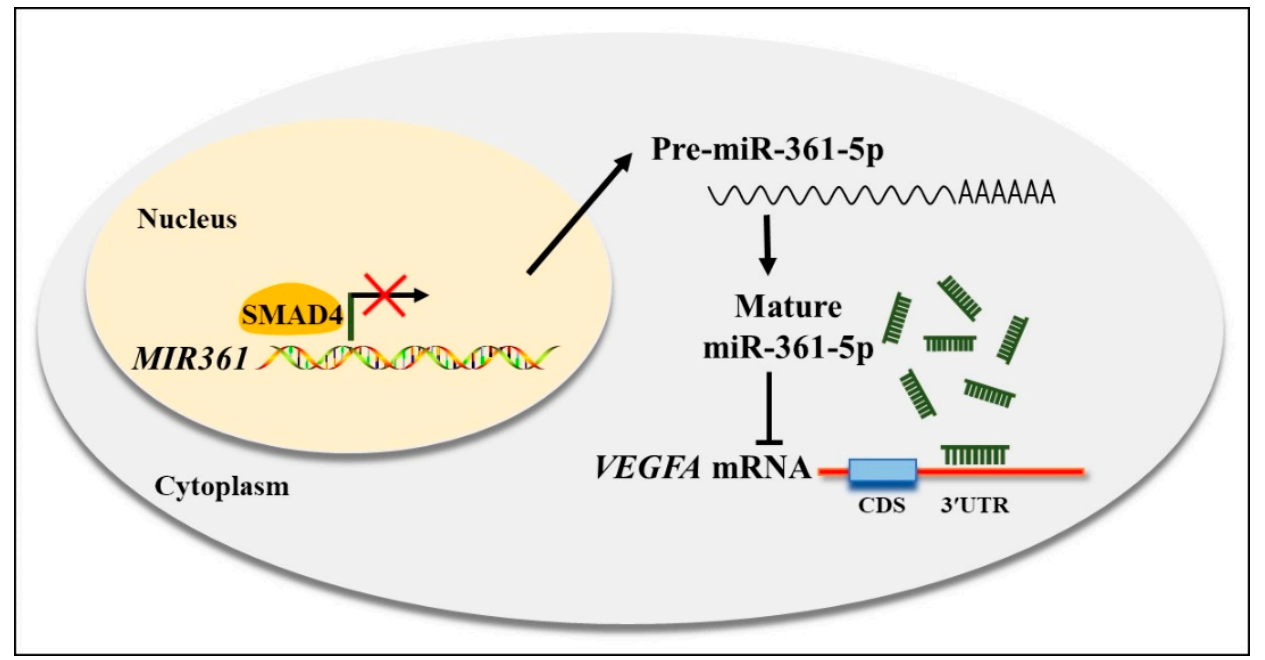

Figure 6. Schematic diagram of SMAD4/miR-361-5p/VEGFA regulatory signaling in porcine GCs. SMAD4 negatively regulates MIR361 transcription by binding to the MIR361 promoter, while matured miR-361-5p reduces VEGFA expression level by directly binding to 3'UTR VEGFA mRNA.

In porcine ovarian granulosa cells, the participation of TGF- $\beta$ signaling has been proved by SMAD4 knockdown [36]. Since then, the apoptosis-inducing capacity of SMAD4 was gradually revealed. Some studies suggested that SMAD4 affects the FSH response because knockdown of SMAD4 significantly inhibited FSH-induced GC proliferation and estradiol production [37]. Additionally, SMAD4 was proven to downregulate miR-143 expression by binding to its promoter, thus opposing the GC apoptosis caused by miR-143 targeting of FSHR [24]. Interestingly, instead of direct binding, the regulatory function of SMAD4 in ovarian granulosa cells is more achieved on the posttranscriptional level through non-coding RNAs, and SMAD4 generally serves as a negative regulator, which resists miRNA transcription, in this case [38]. Our results provide further evidence that SMAD4 could reduce VEGFA levels through miRNA-mediated mechanisms, which adds knowledge to the specific function of TGF- $\beta$ signaling in granulosa cells. The preferences of SMAD4 in miRNA and gene binding and their particularity in different cell types awaits further investigation.

\section{Conclusions}

Our data provide direct evidence that miR-361-5p is upregulated during follicular atresia and that it enhances GC apoptosis by directly targeting the 3'UTR of VEGFA mRNA and downregulating its expression. In addition, TGF- $\beta$ signaling might play a part in VEGFA-mediated GC apoptosis by transcriptional regulation of miR-361-5p expression via its common mediator SMAD4. Overall, our findings broaden the knowledge of VEGFA posttranscriptional regulation in ovarian GC apoptosis and provide novel insights into the mechanism underlying follicular atresia and ovarian physiological function in mammalian ovaries.

Supplementary Materials: The following are available online at http://www.mdpi.com/2218-273X/10/9/1281/s1, Table S1: specific small inference RNA sequences and microRNA sequence are as following, Table S2: qRT-PCR primers were as following, Table S3: Plasmid construction primers are as following.

Author Contributions: Investigation, M.M. and X.G.; formal analysis, M.M., X.G. and W.Y.; writing-original draft preparation, J.Z.; writing-review and editing, Z.P.; supervision, Z.P. and Q.L.; project administration, Z.P.; funding acquisition, J.Z. and Z.P. All authors have read and agreed to the published version of the manuscript.

Funding: This research was funded by the National Natural Science Foundation of China (Grant Nos. 31672421 and 31902123) and the Natural Science Foundation of Jiangsu Province (Grant No. BK20160721). 
Conflicts of Interest: The authors declare no conflict of interest.

\section{References}

1. Marchal, R.; Vigneron, C.; Perreau, C.; Bali-Papp, A.; Mermillod, P. Effect of follicular size on meiotic and developmental competence of porcine oocytes. Theriogenology 2002, 57, 1523-1532. [CrossRef]

2. Manabe, N.; Goto, Y.; Matsuda-Minehata, F.; Inoue, N.; Maeda, A.; Sakamaki, K.; Miyano, T. Regulation Mechanism of Selective Atresia in Porcine Follicles: Regulation of Granulosa Cell Apoptosis during Atresia. J. Reprod. Dev. 2004, 50, 493-514. [CrossRef]

3. Stouffer, R.L.; Martĺnez-Chequer, J.C.; Molskness, T.A.; Xu, F.; Hazzard, T.M. Regulation and Action of Angiogenic Factors in the Primate Ovary. Arch. Med. Res. 2001, 32, 567-575. [CrossRef]

4. Albors, O.L.; Olsson, F.; Llinares, A.; Gutiérrez, H.; Latorre, R.; Candanosa, E.; Guillén-Martínez, A.; Izquierdo-Rico, M. Expression of the vascular endothelial growth factor system (VEGF) in the porcine oviduct during the estrous cycle. Theriogenology 2017, 93, 46-54. [CrossRef] [PubMed]

5. Greenaway, J.; Connor, K.; Pedersen, H.; Coomber, B.L.; Lamarre, J.; Petrik, J. Vascular Endothelial Growth Factor and Its Receptor, Flk-1/KDR, Are Cytoprotective in the Extravascular Compartment of the Ovarian Follicle. Endocrinology 2004, 145, 2896-2905. [CrossRef]

6. Yamamoto, S.; Konishi, I.; Tsuruta, Y.; Nanbu, K.; Mandai, M.; Kuroda, H.; Matsushita, K.; Hamid, A.A.; Yura, Y.; Mori, T. Expression of vascular endothelial growth factor (VEGF) during folliculogenesis and corpus luteum formation in the human ovary. Gynecol. Endocrinol. 1997, 11, 371-381. [CrossRef]

7. Berisha, B.; Schams, D.; Kosmann, M.; Amselgruber, W.; Einspanier, R. Expression and localisation of vascular endothelial growth factor and basic fibroblast growth factor during the final growth of bovine ovarian follicles. J. Endocrinol. 2000, 167, 371-382. [CrossRef]

8. Shimizu, T.; Jiang, J.-Y.; Sasada, H.; Sato, E. Changes of messenger RNA expression of angiogenic factors and related receptors during follicular development in gilts. Boil. Reprod. 2002, 67, 1846-1852. [CrossRef]

9. Bianco, F.; Basini, G.; Grasselli, F. Angiogenic activity of swine granulosa cells: Effects of hypoxia and vascular endothelial growth factor Trap R1R2, a VEGF blocker. Domest. Anim. Endocrinol. 2005, 28, 308-319. [CrossRef]

10. Bianco, F.; Basini, G.; Santini, S.; Grasselli, F. Angiogenic Activity of Swine Granulosa Cells: Effects of Hypoxia and the Role of VEGF. Vet. Res. Commun. 2005, 29, 157-159. [CrossRef]

11. Zhang, J.; Liu, Y.; Yao, W.; Li, Q.; Liu, H.; Pan, Z.-X. Initiation of follicular atresia: Gene networks during early atresia in pig ovaries. Reproduction 2018, 156, 23-33. [CrossRef] [PubMed]

12. Damert, A.; Ikeda, E.; Risau, W. Activator-protein-1 binding potentiates the hypoxia-induciblefactor1-mediated hypoxia-induced transcriptional activation of vascular-endothelial growth factor expression in C6 glioma cells. Biochem. J. 1997, 327, 419-423. [CrossRef] [PubMed]

13. Buteau-Lozano, H.; Ancelin, M.; Lardeux, B.; Milanini, J.; Perrot-Applanat, M. Transcriptional Regulation of Vascular Endothelial Growth Factor by Estradiol and Tamoxifen in Breast Cancer Cells A Complex. Interplay between Estrogen Receptors $\alpha$ and $\beta$. Cancer Res. 2002, 62, 4977. [PubMed]

14. Hanson, J.; Gorman, J.; Reese, J.; Fraizer, G. Regulation of Vascular Endothelial Growth Factor, VEGF, Gene Promoter by the Tumor Suppressor, WT1. Front. Biosci. 2007, 12, 2279-2290. [CrossRef] [PubMed]

15. Chai, Z.-T.; Kong, J.; Zhu, X.-D.; Zhang, Y.-Y.; Lu, L.; Zhou, J.-M.; Wang, L.-R.; Zhang, K.-Z.; Zhang, Q.-B.; Ao, J.-Y.; et al. MicroRNA-26a Inhibits Angiogenesis by Down-Regulating VEGFA through the PIK3C2 $\alpha /$ Akt/HIF-1 $\alpha$ Pathway in Hepatocellular Carcinoma. PLoS ONE 2013, 8, 77957. [CrossRef] [PubMed]

16. Long, J.; Wang, Y.; Wang, W.; Chang, B.H.J.; Danesh, F.R. Identification of MicroRNA-93 as a Novel Regulator of Vascular Endothelial Growth Factor in Hyperglycemic Conditions. J. Boil. Chem. 2010, 285, 23457-23465. [CrossRef]

17. Zhang, L.; Lv, Z.; Xu, J.; Chen, C.; Ge, Q.; Li, P.; Wei, D.; Wu, Z.; Sun, X. Micro RNA -134 inhibits osteosarcoma angiogenesis and proliferation by targeting the VEGFA/VEGFR 1 pathway. FEBS J. 2018, 285, 1359-1371. [CrossRef]

18. Zhao, W.-J.; Zhang, H.-F.; Su, J.-Y. Downregulation of microRNA-195 promotes angiogenesis induced by cerebral infarction via targeting VEGFA. Mol. Med. Rep. 2017, 16, 5434-5440. [CrossRef] 
19. Zhu, X.; Er, K.; Mao, C.; Yan, Q.; Xu, H.; Zhang, Y.; Zhu, J.; Cui, F.; Zhao, W.; Shi, H. miR-203 Suppresses Tumor Growth and Angiogenesis by Targeting VEGFA in Cervical Cancer. Cell. Physiol. Biochem. 2013, 32, 64-73. [CrossRef]

20. Kanitz, A.; Imig, J.; Dziunycz, P.J.; Primorac, A.; Galgano, A.; Hofbauer, G.F.L.; Gerber, A.P.; Detmar, M. The Expression Levels of MicroRNA-361-5p and Its Target VEGFA Are Inversely Correlated in Human Cutaneous Squamous Cell Carcinoma. PLoS ONE 2012, 7, e49568. [CrossRef]

21. Zhou, B.; Ma, R.; Si, W.; Li, S.; Xu, Y.; Tu, X.; Wang, Q.K. MicroRNA-503 targets FGF2 and VEGFA and inhibits tumor angiogenesis and growth. Cancer Lett. 2013, 333, 159-169. [CrossRef]

22. Quezada, M.; Wang, J.; Hoang, V.; McGee, E.A. Smad7 is a transforming growth factor-beta-inducible mediator of apoptosis in granulosa cells. Fertil. Steril. 2012, 97, 1452-1459.e6. [CrossRef] [PubMed]

23. Zhang, H.Y.; Wang, W.; He, Y.; Wang, L.; Tian, K.X.; Song, X.G.; Xu, Y.X. Effects of Silencing Smad4 Gene by Small Interfering RNA on Apoptosis of Porcine Granulosa Cells. Chin. J. Cell Biol. 2010, 32, 596-600.

24. Xing, D.; Zhang, L.; Li, X.; Pan, Z.; Liu, H.; Li, Q. TGF- $\beta$ signaling controls FSHR signaling-reduced ovarian granulosa cell apoptosis through the SMAD4/miR-143 axis. Cell Death Dis. 2016, 7, 2476.

25. Gao, X.; Zhang, J.; Pan, Z.; Li, Q.; Liu, H. The distribution and expression of vascular endothelial growth factor A (VEGFA) during follicular development and atresia in the pig. Reprod. Fertil. Dev. 2020, 32, 259. [CrossRef]

26. Du, X.; Pan, Z.; Li, Q.; Liu, H.; Li, Q. SMAD4 feedback regulates the canonical TGF- $\beta$ signaling pathway to control granulosa cell apoptosis. Cell Death Dis. 2018, 9, 151. [CrossRef]

27. Lin, F.; Li, R.; Pan, Z.X.; Zhou, B.; Yu, D.B.; Wang, X.G.; Ma, X.S.; Han, J.; Shen, M.; Liu, H.L. miR-26b Promotes Granulosa Cell Apoptosis by Targeting ATM during Follicular Atresia in Porcine Ovary. PLoS ONE 2012, 7, e38640. [CrossRef]

28. Wahid, F.; Shehzad, A.; Khan, T.; Kim, Y.Y. MicroRNAs: Synthesis, mechanism, function, and recent clinical trials. BBA-Bioenergetics 2010, 1803, 1231-1243. [CrossRef] [PubMed]

29. Meister, G.; Landthaler, M.; Patkaniowska, A.; Dorsett, Y.; Teng, G.; Tuschl, T. Human Argonaute2 Mediates RNA Cleavage Targeted by miRNAs and siRNAs. Mol. Cell 2004, 15, 185-197. [CrossRef]

30. Zhang, J.; Xu, Y.-X.; Liu, H.; Pan, Z.-X. MicroRNAs in ovarian follicular atresia and granulosa cell apoptosis. Reprod. Boil. Endocrinol. 2019, 17, 9. [CrossRef]

31. Ma, F.; Zhang, L.; Ma, L.; Zhang, Y.; Zhang, J.; Guo, B. MiR-361-5p inhibits glycolytic metabolism, proliferation and invasion of breast cancer by targeting FGFR1 and MMP-1. J. Exp. Clin. Cancer Res. 2017, 36, 158. [CrossRef] [PubMed]

32. Ma, F.; Song, H.; Guo, B.; Zhang, Y.; Zheng, Y.; Lin, C.; Wu, Y.; Guan, G.; Sha, R.; Zhou, Q.; et al. MiR-361-5p inhibits colorectal and gastric cancer growth and metastasis by targeting staphylococcal nuclease domain containing-1. Oncotarget 2015, 6, 17404-17416. [CrossRef] [PubMed]

33. Liu, B.; Lu, B.; Wang, X.; Jiang, H.; Kuang, W. MiR-361-5p inhibits cell proliferation and induces cell apoptosis in retinoblastoma by negatively regulating CLDN8. Childs Nerv. Syst. 2019, 35, 1303-1311. [CrossRef] [PubMed]

34. Shi, X.; Guo, L.-W.; Seedial, S.M.; Si, Y.; Wang, B.; Takayama, T.; Suwanabol, P.A.; Ghosh, S.; DiRenzo, D.; Liu, B.; et al. TGF- $\beta / S m a d 3$ inhibit vascular smooth muscle cell apoptosis through an autocrine signaling mechanism involving VEGF-A. Cell Death Dis. 2014, 5, e1317. [CrossRef] [PubMed]

35. Papoutsoglou, P.; Louis, C.; Coulouarn, C. Transforming Growth Factor-Beta (TGF $\beta$ ) Signaling Pathway in Cholangiocarcinoma. Cells 2019, 8, 960. [CrossRef]

36. Zhang, L.; Du, X.; Wei, S.; Li, N.; Li, Q. A comprehensive transcriptomic view on the role of SMAD4 gene by RNAi-mediated knockdown in porcine follicular granulosa cells. Reproduction 2016, 152, 81-89. [CrossRef]

37. Wang, W.; Chen, X.; Li, X.; Wang, L.; Zhang, H.; He, Y.; Wang, J.; Zhao, Y.; Zhang, B.; Xu, Y.-X. Interference RNA-based silencing of endogenous SMAD4 in porcine granulosa cells resulted in decreased FSH-mediated granulosa cells proliferation and steroidogenesis. Reproduction 2011, 141, 643-651. [CrossRef]

38. Li, Q.; Du, X.; Pan, Z.; Zhang, L.; Li, Q. The transcription factor SMAD4 and miR-10b contribute to E2 release and cell apoptosis in ovarian granulosa cells by targeting CYP19A1. Mol. Cell. Endocrinol. 2018, 476, 84-95. [CrossRef]

(C) 2020 by the authors. Licensee MDPI, Basel, Switzerland. This article is an open access article distributed under the terms and conditions of the Creative Commons Attribution (CC BY) license (http://creativecommons.org/licenses/by/4.0/). 